\title{
The alternative DSM-5 model for personality disorders
}

\section{John M. Oldham}

Menninger Clinic, Baylor College of Medicine, Houston, TX, USA

Differences among personality types and styles have been observed for centuries. What accounts for these differences what makes each person's personality unique - has been debated for a very long time as well. As far back as the days of Hippocrates, it was recognized that there must be correlations between behavior patterns and human biology, and personality types and styles such as melancholic, phlegmatic and sanguine were thought to correlate with differential levels of "body humors" such as bile, phlegm and blood. These principles have stood the test of time, but today we have moved from theory to science and we speak of levels of neurotransmitters such as dopamine, serotonin and norepinephrine that correlate with different personality types and styles. And we recognize that a given individual's personality emerges from at least two sources: temperament (the "hardwired" genetic component) and character (the shaping and molding effects of experience - either healthy or disruptive - during early development, particularly childhood attachment processes).

While great progress has been made, it remains challenging to reach a broad consensus on the best way to classify different personality types, and to differentiate the normal range and variety of personality types from what we call personality disorders. A central feature of this debate has been whether to use a dimensional or a categorical system. The Five-Factor Model has been studied extensively in factor-analytic trait psychology research and has been widely heralded as a valid dimensional system to capture main variations in personality styles (1). This model, however, was derived mostly from studies of normal populations and has not been easily applicable to patient populations.

The DSM adopted a categorical system more compatible with disease classification systems used in the world of medicine. In its third edition, published in 1980, diagnostic criteria were developed that defined a set of eleven personality disorders, and these were placed on the second "axis" (Axis II) of the multi-axial system introduced in that edition of the manual (2). Later editions of the DSM reduced the number of personality disorders to 10 and established a uniform polythetic format for the diagnostic criteria of each disorder, requiring a designated number of criteria to be present to make a given diagnosis (e.g., any 5 of 9 criteria are required for a diagnosis of borderline personality disorder). Personality disorders in DSM-IV are organized in what I refer to as a "dimensionally-flavored categorical system", reflected in the three "cluster" groupings: Cluster A ("odd-eccentric"), Cluster B ("dramatic-emotional"), and Cluster C ("anxiousfearful") (3).
The criteria-defined DSM categorical system has been widely utilized worldwide and has served as a stimulus to research. Nevertheless, a number of problems and shortcomings of this approach have been identified $(4,5)$. For most personality disorders, the number of criteria, or threshold, required to make the diagnosis was arbitrary, yet the categorical approach conveys the impression that the disorder is either present or it is not, rather than that a symptom and trait pattern can vary along a gradient of severity. Furthermore, the polythetic nature of the criteria sets involves extensive heterogeneity within diagnoses. For example, there are 256 ways that five out of nine criteria for the diagnosis of borderline personality disorder can be configured (5), and two patients could receive this diagnosis but share only one criterion.

Work began on DSM-5 over a decade ago, and in an early monograph entitled "A research agenda for DSM-V" it was noted that "well-informed clinicians and researchers have suggested that variation in psychiatric symptomatology may be better represented by dimensions than by a set of categories, especially in the area of personality traits" (6, p. 12). Once convened, the Work Group for Personality and Personality Disorders was charged to review the literature and explore the possibility of developing a dimensional approach to classification of personality disorders.

An initial draft of a prototype model was developed and posted on the DSM-5 website in 2010, along with all proposed changes being considered for DSM-5. After extensive feedback from written responses, professional audiences, and the DSM-5 Task Force itself, it was decided that the prototype model would not be workable. A criteria-based "hybrid" model was then developed and posted in 2011, followed by a final posted version in 2012. This model was studied in the DSM-5 field trials. The new model for borderline personality disorder, for example, showed good testretest reliability (7) and was judged to be preferable to the DSM-IV model by clinicians in routine clinical practice and at academic centers participating in the field trials. Data were obtained (and later published) from an independent group of practitioners showing similar results (8).

The new model for personality disorders was presented to the entire DSM-5 Task Force, consisting of the overall chair and co-chair of the DSM-5 effort, along with the chairs of all of the DSM-5 Work Groups, and it was strongly and unanimously approved. However, several scientific and clinical committees that the American Psychiatric Association (APA) had established to review all proposed changes in the diagnostic manual felt that there was not sufficient 
evidence at the time to validate the proposed new personality disorder model and to establish its clinical utility. The APA Board of Trustees then voted to sustain the DSM-IV diagnostic system for personality disorders, virtually unchanged, in the main section of DSM-5 and to include the proposed new model as an "alternative DSM- 5 model for personality disorders" in Section III of DSM-5, the section referred to as "Emerging measures and models" (9). Although this result was a disappointment to the Work Group, it is encouraging that the new model is included in DSM-5 as an "alternative model", thus "officially" allowing its use by those who are interested, and stimulating research on it (see $5,10,11)$.

In the alternative model, the essential criteria to define any personality disorder are: a) moderate or greater impairment in personality functioning, and b) the presence of pathological personality traits. A "level of functioning" scale is provided, and sensitivity and specificity data supported the designation of "moderate impairment" as the appropriate threshold to indicate the presence of a personality disorder (12). As defined in the alternative model, personality functioning consists of the degree to which there is an intact sense of self (involving a clear, coherent identity and effective selfdirectedness) and interpersonal functioning (reflecting a good capacity for empathy and for mature, mutually rewarding intimacy with others). Pathological personality traits are organized into five trait domains (negative affectivity, detachment, antagonism, disinhibition, and psychoticism), each of which is further explicated by a set of trait facets reflecting aspects of the domain itself. This trait system has been shown to correlate well with the Five Factor Model (13).

One task taken up by the Work Group was to review the literature and assess the strength of the published data supporting the construct validity of each DSM-IV personality disorder, similar to the process carried out in the development of DSM-IV itself, which led to the removal of passiveaggressive personality disorder from the diagnostic manual as a discrete disorder, reconceptualizing it as a trait found in many different Axis I and Axis II conditions. The result of these reviews was to reduce the number of designated personality disorders to six (antisocial, avoidant, borderline, narcissistic, obsessive-compulsive, and schizotypal), and to specify the nature of the moderate or greater impairment in personality functioning, as well as to itemize the pathological personality trait domains and trait facets that characterize each disorder.

In addition, a new diagnosis called Personality DisorderTrait Specified was established, replacing Personality Disorder Not Otherwise Specified in DSM-IV. This diagnosis can now be utilized as more than just a "rule-out" diagnosis - it indicates that a patient does meet the general criteria for a personality disorder, does not qualify for any of the six designated personality disorders, and has a pathological trait profile that can be individually portrayed (which can capture paranoid, schizoid, histrionic, and dependent traits if present, in addition to any other applicable trait facets).
Overall, there has been growing interest in this alternative model. Clinical experience and further research can help evaluate its validity, reliability, and clinical utility, and whether or not additional changes might be considered in future revisions of the diagnostic manual. One interesting model is being proposed for the ICD-11, i.e., to utilize a single diagnostic term of Personality Disorder rated on four levels of personality dysfunction: "personality difficulty" (a "Z" code implying no formal disorder), mild, moderate, and severe personality disorder (14). This proposal is somewhat analogous to the Personality Disorder-Trait Specified diagnosis of DSM-5.

One critique of the alternative model, voiced by a number of leaders in the personality disorder field, argued that the new model is too complicated and that clinicians will not use it (15). However, as described above, clinicians reported favorably on its clinical utility and its use for treatment planning and communication to colleagues, patients, and families. Also, a fair test of complexity is to compare all of DSM-IV personality disorder diagnoses with all of those in the new model. In fact, the number of criteria required to cover all diagnoses in the new model has been reduced by $43 \%$ compared to DSM-IV. Either version can be used prototypically as is common in clinical practice, so that the most prominent diagnostic pattern, such as borderline personality disorder, will command the highest priority in treatment planning, with the option to explore additional pathological features as appropriate.

\section{References}

1. Costa PT Jr, Widiger TA (eds). Personality disorders and the fivefactor model of personality, 2nd ed. Washington: American Psychological Association, 2002.

2. American Psychiatric Association. Diagnostic and statistical manual of mental disorders, 3rd ed. Washington: American Psychiatric Association, 1980.

3. American Psychiatric Association. Diagnostic and statistical manual of mental disorders, 4th ed. Washington: American Psychiatric Association, 1994.

4. Krueger RF, Hopwood CJ, Wright AGC et al. Challenges and strategies in helping the DSM become more dimensional and empirically based. Curr Psychiatr Rep 2014;16:515.

5. Skodol AE. Personality disorder classification: stuck in neutral, how to move forward? Curr Psychiatr Rep 2014;16:480.

6. Rounsaville BJ, Alarcon RD, Andrews G et al. Basic nomenclature issues for DSM-V. In: Kupfer DJ, First MB, Regier DE (eds). A research agenda for DSM-V. Washington: American Psychiatric Association, 2002:1-29.

7. Regier DA, Narrow WE, Clarke DE et al. DSM-5 field trials in the United States and Canada, part II: test-retest reliability of selected categorical diagnoses. Am J Psychiatry 2013;170:59-70.

8. Morey LC, Skodol AE, Oldham JM. Clinician judgments of clinical utility: a comparison of DSM-IV-TR personality disorders and the alternative model for DSM-5 personality disorders. J Abnorm Psychol 2014;123:398-405.

9. American Psychiatric Association. Diagnostic and statistical manual of mental disorders, 5th ed. Washington: American Psychiatric Association, 2013. 
10. Skodol AE, Bender DS, Oldham JM. An alternative model for personality disorders: DSM-5 section III and beyond. In: Oldham JM, Skodol AE, Bender DS (eds). American Psychiatric Press textbook of personality disorders, 2nd ed. Washington: American Psychiatric Publishing, Inc., 2014: 511-44.

11. Krueger RF, Markon KE. The role of the DSM- 5 personality trait model in moving toward a quantitative and empirically based approach to classifying personality and pathology. Annu Rev Clin Psychol 2014;10:477-501.

12. Morey LC, Bender DS, Skodol AE. Validating the proposed Diagnostic and Statistical Manual of Mental Disorders, 5th edition, severity indicator for personality disorder. J Nerv Ment Dis 2013; 201:729-35.
13. Krueger RF, Hopwood CJ, Wright AGC et al. DSM-5 and the path toward empirically based and clinically useful conceptualization of personality and psychopathology. Clin Psychol Sci Pract 2014:21:245-61.

14. Tyrer P. The likely classification of borderline personality disorder in adolescents in ICD-11. In: Sharp C, Tackett JL (eds). Handbook of borderline personality disorder in children and adolescents. New York: Springer, 2014: 451-7.

15. Shedler J, Beck A, Fonagy $\mathrm{P}$ et al. Personality disorders and DSM-5. Am J Psychiatry 2010;167:1026-8.

DOI 10.1002/wps.20232 